Article

\title{
Performance Evaluation of a Vortex Induced Piezoelectric Energy Converter (VIPEC) with CFD Approach
}

\author{
Xinyu An ${ }^{1, \dagger}$, Haocai Huang ${ }^{1,+} \mathbb{D}$, Baowei Song ${ }^{2, \dagger}$ and Congcong Ma ${ }^{3, *, t, \ddagger}$ \\ 1 Ocean College, Zhejiang University, Zhoushan 316021, China; an_xinyu@163.com (X.A.); \\ hchuang@zju.edu.cn (H.H.) \\ 2 School of Marine Science and Technology, Northwestern Polytechnical University, Xi'an 710072, China; \\ songbaowei@nwpu.edu.cn \\ 3 Laboratoire Roberval, Université de Technologie de Compiègne, 60203 Compiègne, France \\ * Correspondence: congcong.ma@utc.fr \\ + These authors contributed equally to this work. \\ ‡ Current address: Marine Science and Technology College, Zhejiang Ocean University, \\ Zhoushan 316022, China.
}

Citation: An, X.; Huang, H.; Song, B.; $\mathrm{Ma}, \mathrm{C}$. Performance Evaluation of a Vortex Induced Piezoelectric Energy Converter (VIPEC) with CFD Approach. Sustainability 2021, 13, 2971. https://doi.org/10.3390/ su13052971

Academic Editor: José Luis Domínguez-García

Received: 31 January 2021

Accepted: 25 February 2021

Published: 9 March 2021

Publisher's Note: MDPI stays neutral with regard to jurisdictional claims in published maps and institutional affiliations.

Copyright: (c) 2021 by the authors. Licensee MDPI, Basel, Switzerland. This article is an open access article distributed under the terms and conditions of the Creative Commons Attribution (CC BY) license (https:/ / creativecommons.org/licenses/by/ $4.0 /)$.

\begin{abstract}
A novel vortex induced piezoelectric energy converter (VIPEC) was present in this paper to harvest flow kinetic energy from the ambient environment through a piezoelectric beam. The converter consists of a circular cylinder, a pivoted beam attached to the tail of the cylinder and several piezoelectric patches. Vortex induced pressure difference acts on the beam and drives the beam to squeeze piezo patches to convert fluid dynamic energy into electric energy. Transition Shear Stress Transport (SST) combined with Scale Adaptive Simulation (SAS) model was employed to predict the turbulent flow and flow separation around the cylinder with various beam lengths at high Reynolds number of $8 \times 10^{4}$ based on the computational fluid dynamics (CFD) approach. The accuracy of SST-SAS model was investigated through verification and validation studies. The output voltage equation was derived from the piezoelectric constitutive equation. It was revealed that the beam length influences the flow wake pattern, the separation angle and shedding frequency greatly through changing the adverse pressure gradient around the cylinder. The wake pattern becomes symmetrical about the beam when the beam length is longer than a critical value. The length of the beam has little influence on the separation angle. When the beam length is about 1.3 times the diameter of the cylinder, the shedding frequency and output voltage achieves its maximum, and the separation angle is minimal. Maximal output voltage reaches $20 \mathrm{mV}$.
\end{abstract}

Keywords: transition SST-SAS; high Reynolds number flow; separation angle; shedding frequency

\section{Introduction}

Over the past few decades, many researches have been focused on obtaining electrical energy from the ambient environment. Taking advantage of the piezoelectric effect, piezoelectric energy converters have been used to absorb ambient energy, which enables a greater conversion rate of ambient mechanical energy to electrical energy [1]. The cantilever beam is one of the most commonly used structures in piezoelectric energy harvesters for low frequency applications [2], especially in a fluid environment.

Taylor et al. [3] developed a so called 'energy harvesting eel', which is composed of a long strip of piezoelectric polymer bimorph material and a bluff body placing in its upstream. Periodically shedding vortices from either side of the obstruction result in pressure difference over the strip that causes the piezoelectric bimorph to 'wave', which is similar to the motion of an eel. Pobering and Schwesinger [4] conducted researches on the ability of a PVDF (Polyvinylidene Fluoride) flag, similar to the eel created by Taylor et al. [3]. Wind energy harvesters using PVDF have also been studied. PVDF films were used as a cantilever [5,6] or attached to a leaf-shaped structure [7]. Akaydin et al. [8] combined 
piezoelectric energy harvester with vortex induced vibration (VIV), which was a novel form to utilize flow kinetic energy and based on which, various kinds of devices [9-11] had been developed both under water and in the air. Akaydin et al. experimented the wake of a circular cylinder through the output voltage of a piezoelectric cantilever beam, in which the beam is parallel to the incoming flow and cantilevered at its downstream end. He concluded that maximum power output was measured when the tip of the beam is about two diameters downstream of the cylinder. In his later work [12], he experimented a self-excited piezoelectric harvester subjected to a uniform and steady flow. The harvester consists of a cylinder connected to the free end of a cantilever beam and piezoelectric patches partially covering the clamped end. Researches on multilayer piezoelectric beam were performed as well. Dai et al. [13] also investigated a similar multi-layered piezoelectric cantilever beam with a circular cylinder tip mass attached to its end which is placed in a uniform air flow and subjected to direct harmonic excitations. An et al. [14] modeled the transverse motion of a bluff body and blending of a tri-layered piezoelectric cantilever beam based the lift decomposition model and Euler-Bernoulli assumption. The beam's deflection and generated voltage were obtained for an anchor system. Pan et al. [15] used a modified circular cylinder with a slit and concave rear surface to produce stronger vortices. A PVDF cantilever beam was positioned along the centerline of the vortex shedder to harvest the vibration energy from swirling vortices. He proved taht the effectiveness and the harvested energy was improved with the modified harvesting system. Hai et al. [16] adopted a 2-DOF (Degree of Freedom) piezoelectric harvester to collect a broadband underwater flow induced vibration energy. He found that the system using 2-DOF harvester can achieve higher electric energy than that using a Single-DOF harvester. Sun and Su [17] presented a 2-DOF piezoelectric energy for scavenge wind VIV energy, which was composed of an outer beam, an inner beam, a cylinder and tip masses attached to the beams. His experimental results showed that the 2-DOF design achieves higher energy than that of the 1-DOF counterpart at both low speed and high speed condition. Yang and He [18] considered the effect of the piezoelectric layer to lower the cut-in speed of VIV-galloping interactive piezoelectric wind energy harvester. The side length of the bluff body's crosssection was used to control a behavior transition from separated to interacted VIV and galloping. Zhou et al. [19] utilized a piezoelectric bimorph with a pulling strip to collect wind VIV energy from a rotating machinery. He revealed that with a certain piezoelectric bimorph, choosing a installing angle can increase the system's power generation capacity more effectively. Hou et al. [20] introduced the nonlinear magnetic force into the harvester and developed a nonlinear monostable magnetic-coupling piezoelectric energy harvester. Its performance with the used PZT length was investigated based on the Euler-Bernoulli beam theory. Kundu and Ghosal [21] studied the plate deformation controlled by a bluffbody located in front of a flexible plate at Reynolds number is 100 . He demonstrated the feasibility of harvesting energy from pulsatile flow by using a bluff body.

However, despite various devices mentioned above, based on the author's knowledge, seldom research study has been conducted combining vortex induced vibration and the piezoelectric beam with the existing underwater equipment or resident platforms. The present work investigates a novel vortex induced piezoelectric energy converter (VIPEC), which consists of a circular cylinder and a pivoted beam attached the tail of the cylinder and several piezo patches fixed inside the cylinder. The converter's output voltage equation was derived from the piezoelectric equation. Numerical simulations were conducted to investigate the influence of the beam length on flow pattern, the fluid parameters such as the shedding frequency and the separation angle. The output voltage was obtain from the derived equation and calculated lift force on the beam.

\section{Description of the VIPEC}

The VIPEC is mainly composed of a circular cylinder bluff body, a pivoted beam and several piezoelectric patches, as shown in Figure 1a. The bluff body can be the anchor chain and is deemed to connect with a base such as seabed or its floating platform. Piezoelectric 
patches are amounted on either side of the beam inside the construction in order not to influent the vortex shedding on the cylinder. The rotation axis of the pivoted beam is also inside of the cylinder, ahead of the piezoelectric patches, which are essential to press them for voltage output. Two mechanisms, i.e., the impingement of induced flow of shedding vortices on one side of the beam and the low pressure core region of vortices present at the opposite side of the beam contribute the driving force, which drives the beam to squeeze its adjacent piezo patches to generate voltage. This converter is intended to be utilised in an underwater environment, such as covering the anchor chain of resident platforms.

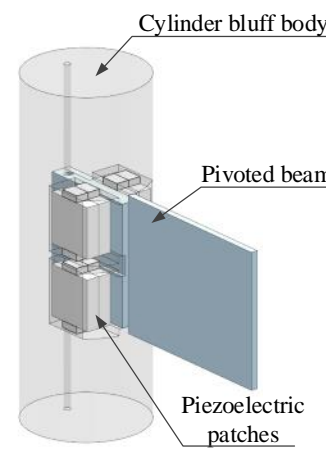

(a)

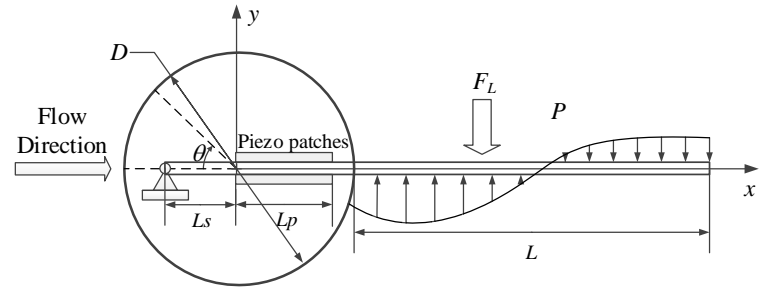

(b)

Figure 1. The designed VIPEC: (a) the geometric diagram, (b) the simplified physical model.

\section{Simplified Physical Model}

Rigid material PVC is adopted for the attached beam, and its deformation can be ignored in comparison with its thickness. Since straight pivoted beam is utilized in the wake, the converter has the same geometry in any cross-section along the beam's height. Given the span length does not affect the converter's performance, a two-dimensional analysis was adopted in the present study. In two-dimensional simulations, Figure $1 \mathrm{~b}$ illustrates the force diagram of a simplified physical model of VIPEC due to vortex shedding. The cylinder's center is selected as the origin of coordinates. $\theta$ is the azimuth angle around the cylinder. $P$ is the distributed pressure on the beam, which is just a randomly curved pressure distribution. $D$ is the diameter of the cylinder.

\section{Numerical Method}

The two-dimensional numerical simulations presented in this paper were carried out on commercial code ANSYS Fluent 17.0, which is based on the finite volume method [22].

\subsection{Governing Equation}

Navier-Stokes equations are the governing equation of fluid computation. In this paper, the transition shear stress transport (SST) turbulence model combined with ScaleAdaptive Simulation (SAS) developed by Egorov and Menter was adopted to solve incompressible unsteady Reynold-averaged Navier-Stokes (URANS) equations and to predict the turbulent flow in transient simulations at high Reynolds number flows. The SAS concept is based on the introduction of the von Kármán length-scale into the turbulence scale equation, which allows the SAS model to dynamically adjust to resolved structures and results in LES-like behaviours in unsteady regions of the flow field.

31-type piezoelectric patches were employed in the converter, which means the direction of polarization is perpendicular to that of applied stress. The open circuit output voltage $v$ was derived from the piezoelectric constitutive equation, and is written as:

$$
\frac{d v(t)}{d t}-\frac{h_{p}}{\varepsilon_{33}^{T} t_{p} L_{p} R_{l}} v(t)=-\frac{d_{31} h_{p} L_{\text {ratio }}}{\varepsilon_{33}^{T} t_{p} L_{p}} \frac{d F_{l}(t)}{d t}
$$

where, $h_{p}$ is the thickness of piezo patches, $t_{p}$ is the height of piezo patches, $L_{p}$ is the width of piezo patches, $\varepsilon_{33}^{T}$ is the permittivity electric constant, $d_{31}$ is the piezoelectric constant, 
$L_{\text {ratio }}$ is the ratio of the arm of lift force on the beam to that of the normal stress acting on piezo patches, $R_{l}$ is the load resistance, $F_{L}$ is the lift force acting on the beam and $L_{s}$ is the distance between the rotation axis and piezo patches.

\subsection{Computational Domains and Grid Generation}

The computational domain had a width of $20 D$ and a length of $40 D$ (as showed in Figure 2), where $D$ refers to the diameter of the bluff body. The converter was placed in the symmetry of the top and bottom and at a distance of $15 D$ from the inlet boundary. Boundary conditions employed consisted of a velocity inlet on the left side; a pressure outlet on the right side and two slipping walls on the top and bottom sides.

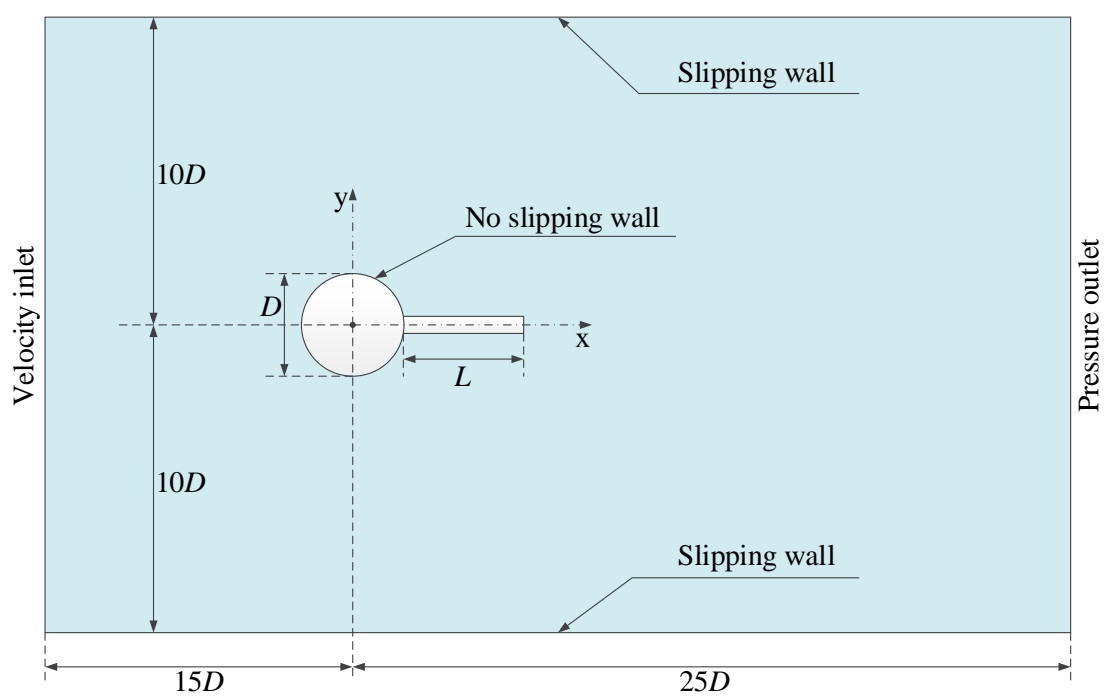

Figure 2. The two-dimensional computational domain and boundary conditions for numerical simulations.

The entire domain was discretized with quadrilateral elements. Grids closest to the profiles of the converter were refined with rectangular elements to describe the boundary layer. A maximum layers of 20 is set to capture the boundary layer flow in the model's near-wall zone. The first layer thickness is $0.005 \mathrm{~mm}$ and the growth ratio is set as 1.1. The final grid is given in Figure 3.

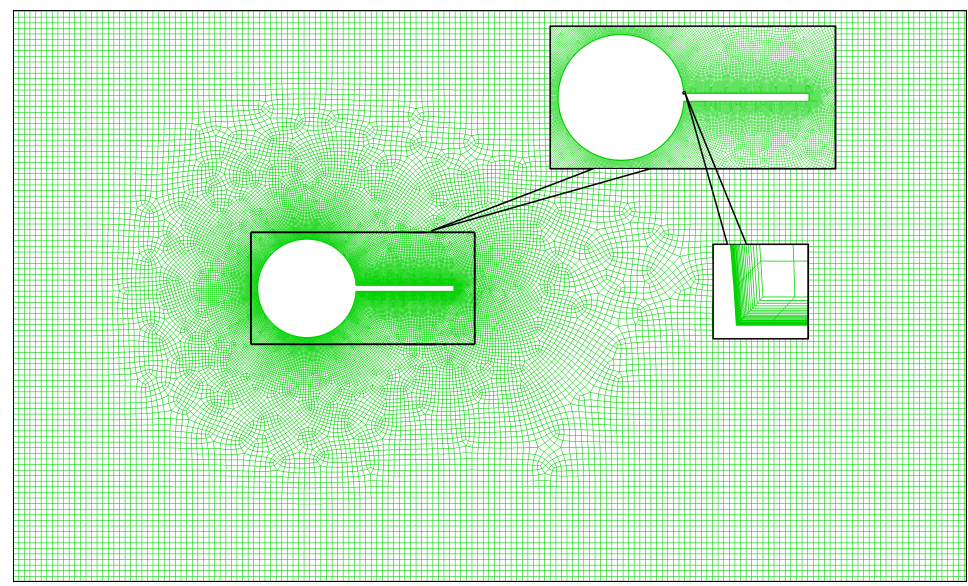

Figure 3. Computational grid used for all simulations and close-up views around the model and its boundary layers. 


\subsection{Solution Setups}

Multiple simulations were carried out for various beam lengths, and the dimensionless beam length $L^{*}=L / D$ was set as $1,1.2,1.3,1.4,1.7,2$ at $R e=\frac{U D}{v}=8 \times 10^{4}$, where $U$ is the free flow inlet velocity, $v$ is the dynamic viscosity. Convergences were determined by the order of magnitude of the governing equations' residuals. The criteria of all scaled residuals including continuity equation, momentum equation, $k$ equation and $\omega$ equation below $1 \times 10^{-5}$ were employed as the convergence criterion in order to get reliable results. The timestep was set as $0.001 \mathrm{~s}$ according to Courant number and the maximal iterations of each time step were set as 100, which enabled all the residuals to meet convergence in all simulation cases. The coupled pressure-velocity coupling method was employed in all simulations. A second-order upwind spatial discretization algorithm was used for all the equations, including pressure, momentum and turbulence, and a least squares cell based algorithm was used for the gradients. The convection term and turbulence term were both discretized by the second-order upwind difference scheme in the present study. The second order backward Euler scheme was employed to discretize the transient term. The second-order algorithm is more accurate than first order one because it reduces interpolation errors and false numerical diffusion.

\section{CFD Method Verification and Validation}

\subsection{Verification}

In the convenience of comparing with other's simulation and experimental results, numerical verification and validation in this paper were processed under flow past a smooth cylinder at $R e=1 \times 10^{6}$. A grid convergence study was performed to evaluate the influence of grid resolution on the lift coefficient $C_{L}$, the drag coefficient $C_{D}$, and Strouhal number $S_{t}$. Three different grid resolutions, whose total elements were controlled by max face size, and their calculated results are listed in Table 1. Because most elements were aggregated in the neighbourhood of the model, results of medium and fine grid cases had little difference. It can be noted that medium and fine girds gave substantially the same results and agree well with Large Eddy Simulation (LES) results given by Catalano et al. [23]. and published experimental data [24]. Considering time consumption in the simulation, the medium grid resolution was adopted in the following researches.

Table 1. Numerical verification with three different grid resolutions and relevant results.

\begin{tabular}{cccccc}
\hline Mesh Resolution & Max Face Size & Total Elements & $C_{L}$ & $C_{D}$ & $S_{t}$ \\
\hline Coarse & 60 & 68,000 & 0.081 & 0.40 & 0.268 \\
Medium & 30 & 70,000 & 0.090 & 0.35 & 0.323 \\
Fine & 15 & 89,000 & 0.092 & 0.33 & 0.327 \\
LES & - & - & - & 0.31 & 0.35 \\
Published experimental data & - & - & $0.21-0.63$ & $0.17-0.40$ & $0.18-0.50$ \\
\hline
\end{tabular}

\subsection{Validation}

In order to validate the accuracy of the turbulent model adopted in this paper, calculated pressure coefficient around the cylinder at $R e=1 \times 10^{6}$ is showed in Figure 4. It can be found that the simulation result agrees well with that of LES and Falchabart's [24] and Warschauer and Leene's experiments [25], which means reliable results of this issue can be obtained from the adopted turbulent model. 


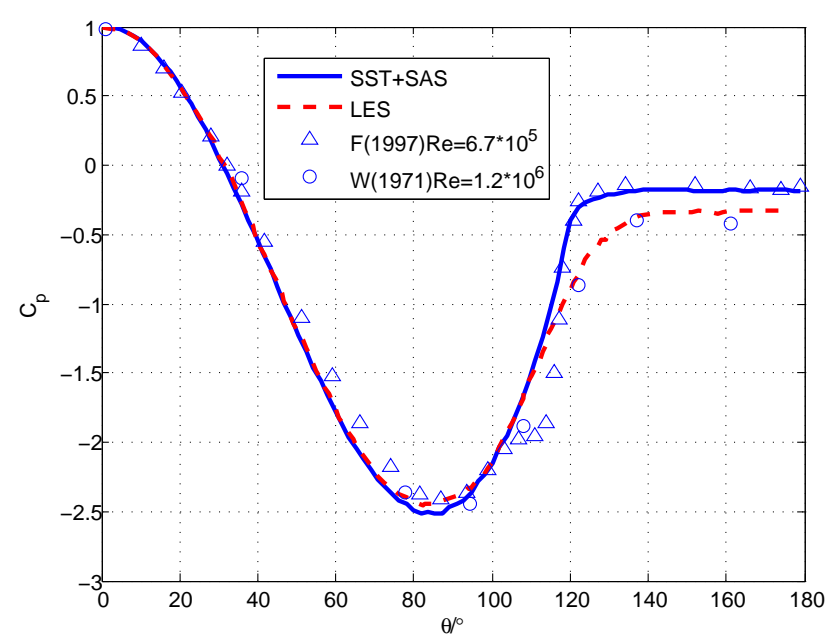

Figure 4. Pressure distribution on the cylinder.

\section{Results and Discussion}

\subsection{Effect of the Beam Length on Wake Region}

Vortex sheds periodically in the wake of a circular cylinder in the region of the critical Reynolds number [26]. When a beam is attached behind the cylinder, the formation and shedding of a vortex is severely affected and inhibited after the separation point around the cylinder. As a consequence of counter flow alongside the beam caused by the negative pressure core region, a dominated large scale vortex is formed over each side of the beam. When the beam is sufficiently short $L^{*}=1$, shedding vortex slips alongside the beam and vortices over both sides aren't formed simultaneously, as showed in Figure 5. It can develop downwards along the beam across its centerline. Besides a dominated scale vortex (labeled as 'A2' in Figure 5), no coherent vortical structure can be discovered. Whereas, the pressure difference between the beam's each side exists as well owing to the asynchronous flow separation around the two separation regions, then distributed force is produced over the beam because of the pressure difference. However, due to the limitation of the beam length, lift force doesn't accumulate to its maximum.

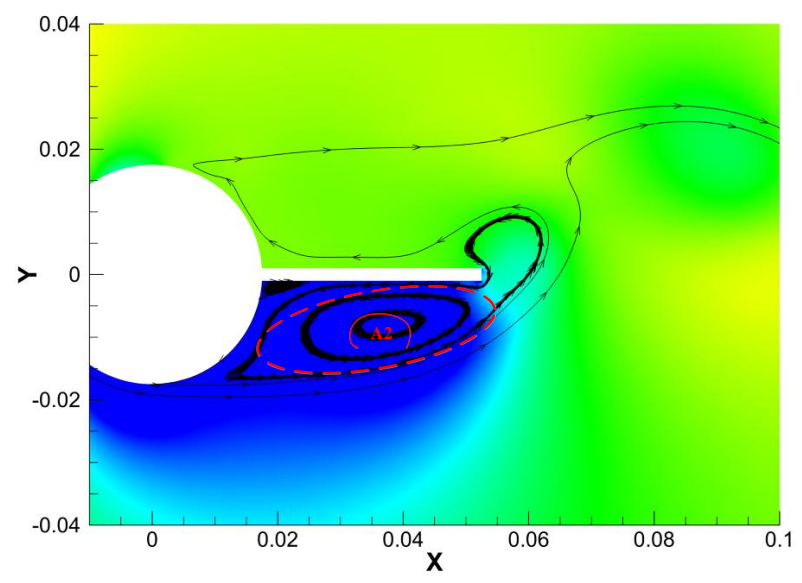

Figure 5. Pressure contour and negative pressure core around the beam for $L^{*}=1$.

When the beam length becomes longer, the dominated large scale vortex continues slipping along the whole beam. However, another reverse large scale vortex sheds on the other side of the cylinder before the previous vortex detaches from the beam. The development of the dominated large scale vortex over one side of the beam during half of a shedding period is shown in Figure 6. 

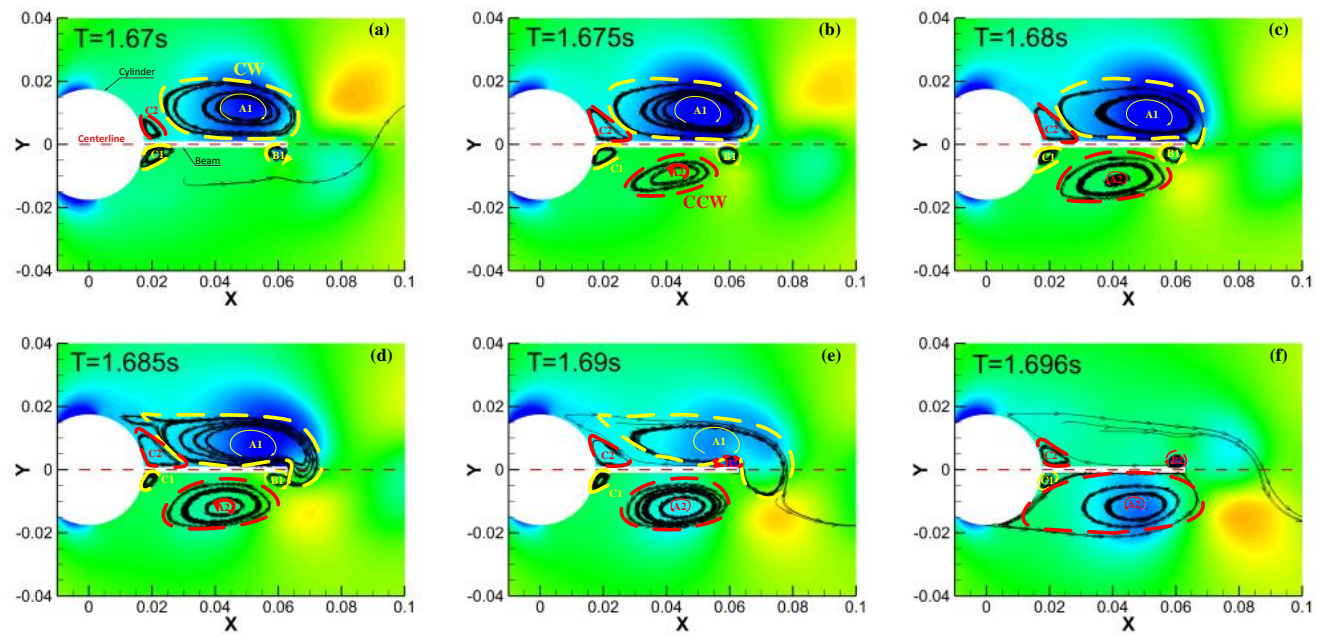

Figure 6. Pressure contour and negative pressure core around the beam for $L^{*}=1.3$ (Dashed lines in yellow and the label ' 1 ' means clockwise $(\mathrm{CW})$, dashed lines in red and the label ' 2 ' means counterclockwise (CCW)).

As it can be seen in Figure 6, a clockwise (CW) dominated large scale vortex (labeled as 'A1' in Figure 6 in yellow) slips along the beam and cross its centreline (Figure 6a). Meanwhile, a counterclockwise (CCW) vortex (labeled as 'A2' in Figure 6 in red) is formed and slips over the other side of the beam (Figure $6 \mathrm{~b}$ ). When 'A1' meets ' $\mathrm{A} 2$ ', it is squeezed to detach from the beam, swims and dissipates downstream. A small vortex (labeled as ' $\mathrm{C} 1$ ' and ' $\mathrm{C} 2$ ' in Figure 6) exists at the both corners behind the cylinder. Both vortices ' $\mathrm{C} 1$ ' and ' $\mathrm{C} 2$ ' swirl in the opposite direction of its neighboring vortex, and they are induced by the counter flow of vortices 'A2' and 'A1' separately. Therefore, their vorticity is characterized by 'A2' and 'A1'. When 'A1' reaches the upper free corner, it moves across the centerline with an angle to the beam end rather than parallel to or along it (Figure 6c) due to the sharp/rectangular angle of the corner. Once the reflux moves lower than the upper side, flow separation also occurs near this corner. As a result of flow separation, the near-wall flow at the upper end corner tends to deviate the beam's surface (Figure $6 \mathrm{~d}$ ). Hence a vortex in the reverse direction (labeled as 'B2') is forming in Figure 6e. In fact, the formation of vortex 'B2' is formed for the same mechanism of ' $\mathrm{C} 2$ ' and similarly, both clockwise vortices ' $\mathrm{C} 1$ ' and 'B1' are induced by the counterclockwise vortex 'A2'. When 'A1' reaches the lower free end of the beam, it 'swallows' vortex 'B1' (Figure 6d,e) and then it detaches form the free end and flows downwards. Meanwhile, the counterclockwise vortex 'B2' is formed and 'A2' is dominating over the lower side the beam (Figure 6f), which is just the opposite contour view of it in Figure 6a.

With even stretched beam, saying $L^{*}=2$ vortices 'A1' and 'A2' dominate over each entire side of the beam simultaneously. However, vortices 'B1', 'B2', 'C1', and 'C2' aren't induced since shedding vortices 'A1' and 'A2' don't move across the centerline when they detach from the beam, as seen in Figure 7. Therefore, tender driving force is exerted on the beam as a result of the pressure difference's reduction and vortex shedding around the cylinder is severely inhibited. 


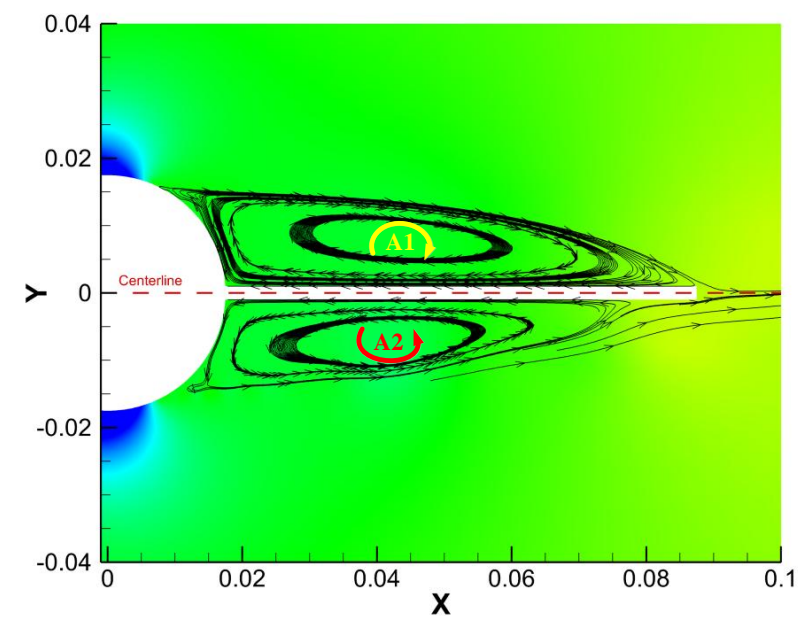

Figure 7. Pressure contour and negative pressure core around the beam for $L^{*}=2$.

\subsection{Influence of the Beam Length on Fluid Parameters}

The beam's lift force and moment variations of $L^{*}=1.7$ are shown in Figure 8a during $19.9 \mathrm{~s}$ to $20.1 \mathrm{~s}$. Dividing moment of force by the corresponding lift force, the center of pressure at each moment will be acquired. It is interesting to notice that the pressure center shifts periodically (as shown in Figure 8b) and it can become rather insignificant when total lift force approaches to 0 . This shift demonstrates the movement and development of shedding vortices along the beam. Finally, using fast Fourier transform (FFT), the fluctuating frequency of all above lift and moment reads $16.55 \mathrm{~Hz}$ (as shown in Figure 8c), and it is also the shedding frequency. The equivalent analysis process procedure for $L^{*}=1.7$ has been carried out on all other simulation cases.

Flow separation occurs at the point where $\frac{\partial u}{\partial y}=0$ and flow separation angle can be calculated from this criterion. Once a beam is appended to the rear of the cylinder, it affects, even destroys the cylinder's wake field. As a consequence, the wake flow influences the separation point and the change of the separation point has an influence on the wake region vice versa. The variation of the separation point around the cylinder due to its affiliated beam is shown in Figure 9. With the increase of the beam length, separation angle decreases and reaches its minimum at $L^{*}=1.3$. The forward shift of the separation point in $L^{*}=1.3$ reflects the shedding vortices' development along the beam. These vortices decrease the intensity of adverse pressure gradient on the afterbody of the cylinder, which leads to the kinetic energy of fluid be capable of overcoming adverse pressure gradient and viscous effects on longer distance around the cylinder, thus vortices shedding occurs at a more backward position. Then, with the increase of the beam length, vortices at each side of the beam are more likely to have a similar pattern, the adverse pressure gradient is not that strong and corresponding separation angle rises, which means weaker vortex shedding. It is reasonable to find that separation angles of all cases are bigger than that of the smooth cylinder case (without an beam, nearly $85^{\circ}$, shown as a blue dash line in Figure 9). What's more, the fluctuation of separation angles is quite small (within $7^{\circ}$ ), which means the beam's length has little influence on the separation angle. This is in corresponding with Grove et al.'s experimental results [27].

The curved change of the shedding frequency calculated from processing FFT on the corresponding lift force is also shown in Figure 9. It is in an opposite tendency with the separation angle. It first rises and reaches at $L^{*}=1.3$. Then it decreases when the beam's length grows. However, no matter how it varies, the shedding frequency of a cylinder with an attached beam retains less than that of the smooth cylinder (nearly $17.84 \mathrm{~Hz}$, shown as a red dash line in Figure 9), which is also a consequence of the decrease of adverse pressure gradient at the afterbody of the obstruction just like that of separation angle. 


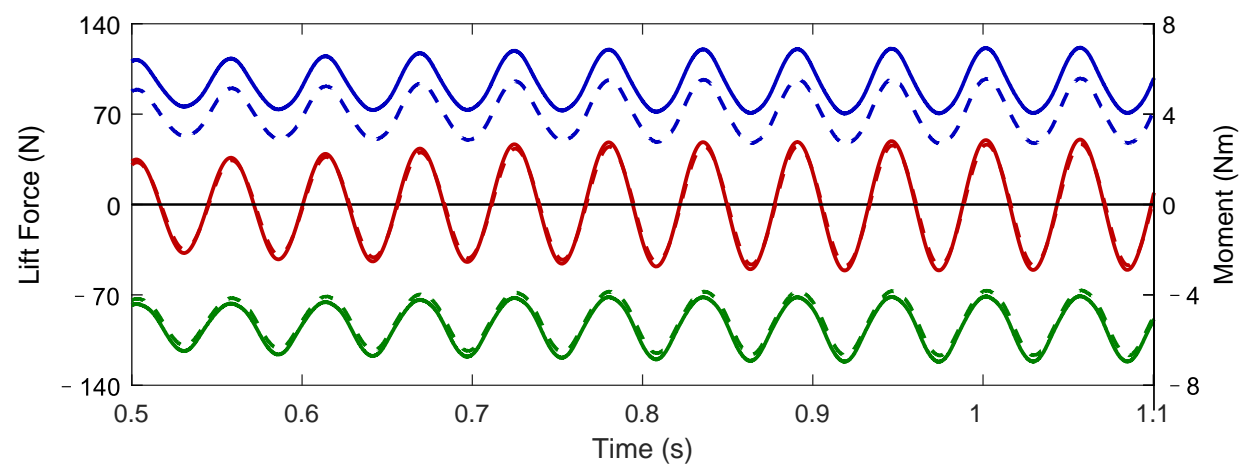

(a)

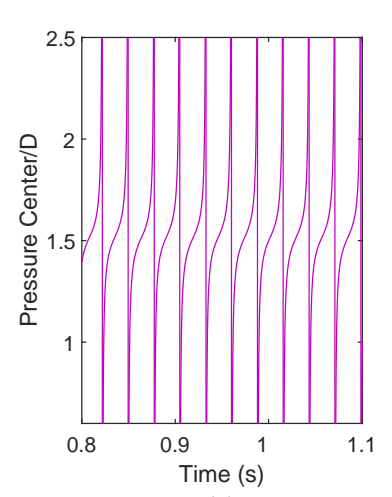

(b)

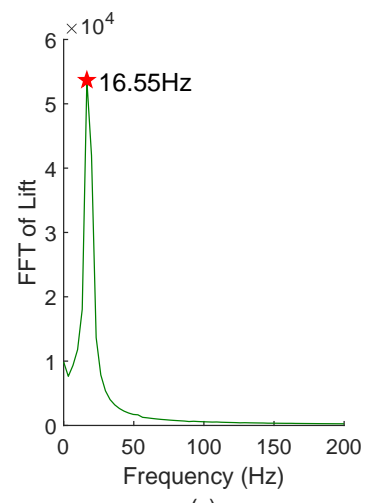

(c)

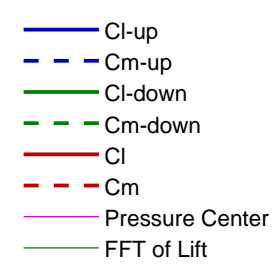

Figure 8. Results processing for $L^{*}=1.7$ : (a) $C_{L}$ and $C_{m}$ for the upside and downside of the beam and the entire beam; (b) The variation of the dimensionless lift force center; (c) Fast Fourier Transform of $C_{L}$ and corresponding shedding frequency.

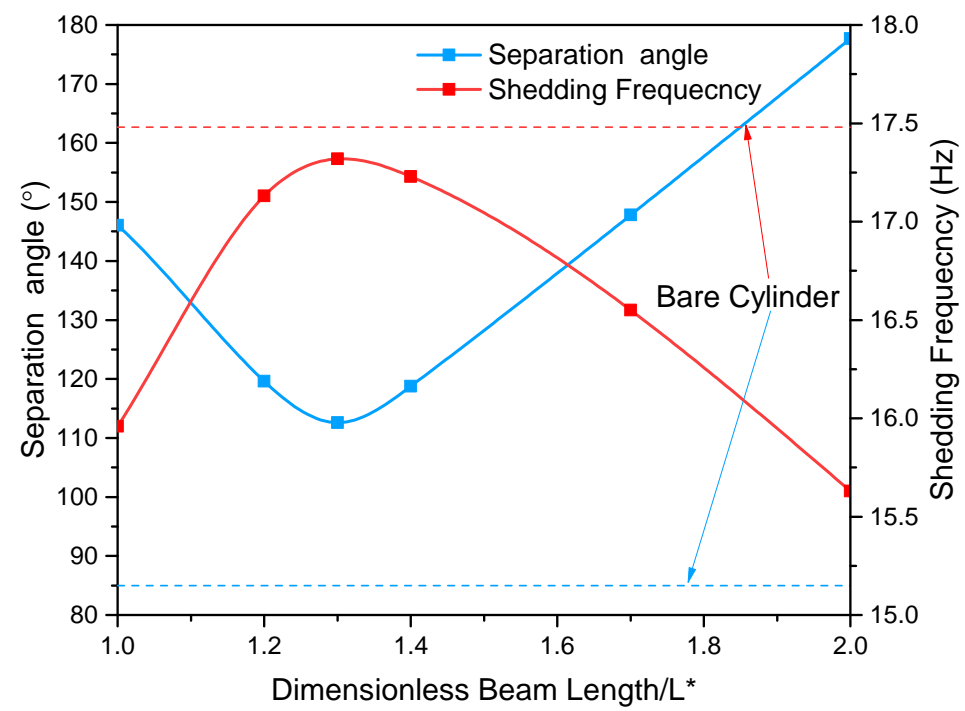

Figure 9. Flow separation angle and vortex shedding around the cylinder of different simulation cases.

\subsection{Influence of the Beam Length on the Converter's Performance}

The beam moves back and forth periodically as a result of the exerted lift force and momentum. Fluid kinematic energy is converted into the beam's mechanical energy, which is also a source of harvested electric power, through the movement of the beam in any direction. Utilizing obtained lift forces on the beam, the converter's output voltage can be calculated from (1). The variation of the output voltage with respect to the dimensionless beam length is illustrated in Figure 10. Obviously, power transferred to the beam grows with the increase of the beam's length. However, when the beam is greater than a critical 
value ( $L^{*} \approx 1.3$ ), the transferred energy decreases dramatically. These simulations demonstrate that with the beam's dimensionless length equal $1.3\left(L^{*} \approx 1.3\right)$, the output voltage peak reaches around $20 \mathrm{mV}$. The output voltage becomes very small for $L^{*}>2$.

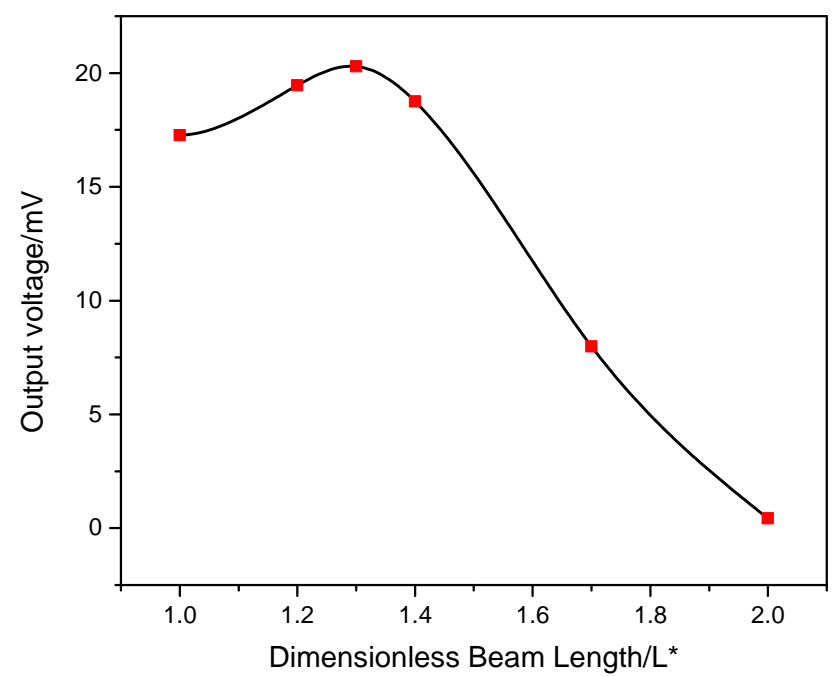

Figure 10. The variation of open circuit output voltage with respect to the dimensionless beam length.

\section{Conclusions}

In this study, a novel form of vortex induced piezoelectric energy converter based on vortex shedding in fluid flows was presented. Utilizing a simplified physical model, two-dimensional transient simulations were computed adopting combined transition SST and SAS (SST-SAS) turbulence model. Three different grid resolutions were employed in the verification and validation study. The output voltage equation was derived using 31-type piezoelectric patches from the piezoelectric constitutive equation. Based on the computational results, the influence of the beam's length on the wake field, separation angle and shedding frequency were investigated, and the output voltage was obtained. The following conclusions are drawn as follows:

(a) With a beam attached to the rear of a cylinder, both the separation angle and the vortex shedding frequency become greater than that of the smooth cylinder. The beam's length does not have much influence on the separation angle.

(b) The development of shedding vortices alongside the beam affects the adverse pressure gradient around the cylinder. Thus, the beam length has a great effect on the flow wake pattern, the separation angle and the shedding frequency. When the attached beam is sufficiently long, saying $L / D>2$, the flow pattern tends to become symmetrical about the beam.

(c) Due to the secondary separation of the dominant large scale vortex ' $\mathrm{A}$ ' at the free end of the beam, the case of $L / D=1.3$ under $R e=8 \times 10^{4}$ have a relative maximum of the vortex shedding frequency, minimal separation angle and maximal estimated output voltage energy as well. The maximal output voltage reaches $20 \mathrm{mV}$.

Author Contributions: Conceptualization, X.A.; validation, C.M. and X.A.; formal analysis, X.A. and C.M.; investigation, X.A. and C.M.; data curation, X.A.; writing-original draft preparation, C.M.; writing-review and editing, X.A.; visualization, C.M. and X.A.; supervision, B.S. and H.H.; project administration, B.S.; funding acquisition, X.A., H.H. and B.S. All authors have read and agreed to the published version of the manuscript.

Funding: This research was funded by the National Natural Science Foundation of China (Grant No. 51179159) and Zhejiang Provincial Postdoctoral Science Foundation (Grant No. ZJ2020137).

Data Availability Statement: Not appliable.

Conflicts of Interest: The authors declare no conflict of interest. 


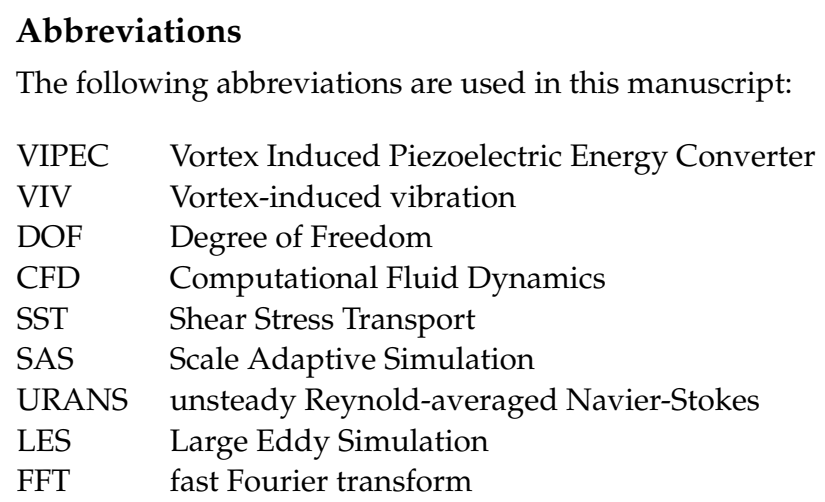

\section{References}

1. Anton, S.R.; Sodano, H.A. A review of power harvesting using piezoelectric materials (2003-2006). Smart Mater. Struct. 2007, 16. [CrossRef]

2. Li, H.; Tian, C.; Deng, Z.D. Energy harvesting from low frequency applications using piezoelectricmaterials. Appl. Phys. Rev. 2014, 1, 041301. [CrossRef]

3. Taylor, G.W.; Burns, J.R.; Kammann, S.M.; Powers, W.B.; Welsh, T.R. The Energy Harvesting Eel: A Small Subsurface Ocean/River Power Generator. IEEE J. Ocean. Eng. 2001, 26, 539-547. [CrossRef]

4. Pobering, S.; Schwesinger, N. A novel hydropower harvesting device. In Proceedings of the International Conference on MEMS, NANO and Smart Systems, Banff, AB, Canada, 25-27 August 2004; pp. 480-485. [CrossRef]

5. Li, S.; Yuan, J.; Lipson, H. Ambient wind energy harvesting using cross-flow fluttering. J. Appl. Phys. 2011, 109, 109-111. [CrossRef]

6. Vatansever, D.; Hadimani, R.L.; Shah, T.; Siores, E. An investigation of energy harvesting from renewable sources with PVDF and PZT. Smart Mater. Struct. 2011, 20, 55019-55024. [CrossRef]

7. Oh, S.J.; Han, H.J.; Han, S.B.; Lee, J.Y.; Chun, W.G. Development of a tree-shaped wind power system using piezoelectric materials. Int. J. Energy Res. 2010, 34, 431-437. [CrossRef]

8. Akaydin, H.D.; Elvin, N.; Andreopoulos, Y. Wake of a cylinder: A paradigm for energy harvesting with piezoelectric materials. Exp. Fluids 2010, 49, 291-304. [CrossRef]

9. Bernitsas, M.M.; Raghavan, K.; Ben-Simon, Y.; Garcia, E.M.H. VIVACE (Vortex Induced Vibration for Aquatic Clean Energy): A New Concept in Generation of Clean and Renewable Energy from Fluid Flow. J. Offshore Mech. Arctic Engineering-Trans. ASME 2008, 130, 619-636. [CrossRef]

10. Bernitsas, M.M.; Ben-Simon, Y.; Raghavan, K.; Garcia, E.M.H. The VIVACE Converter: Model Tests at High Damping and Reynolds Number Around 105. J. Offshore Mech. Arct. Eng. 2009, 131, 403-414. [CrossRef]

11. Vinod, A.; Kashyap, A.; Banerjee, A.; Kimball, J. Augmenting Energy Extraction from Vortex Induced Vibration Using Strips of Roughness/Thickness Combinations. Available online: http://marineenergytechnologysymposium.org/download/2013 / AUGMENTING-ENERGY-EXTRACTION-FROM-VORTEX-INDUCED.pdf (accessed on 22 July 2015).

12. Akaydin, H.D.; Elvin, N.; Andreopoulos, Y. The performance of a self-excited fluidic energy harvester. Smart Mater. Struct. 2012, 21, 25007-25019. [CrossRef]

13. Dai, H.L.; Abdelkefi, A.; Wang, L. Piezoelectric energy harvesting from concurrent vortex-induced vibrations and base excitations. Nonlinear Dyn. 2014, 77, 967-981. [CrossRef]

14. An, X.; Song, B.; Mao, Z.; Ma, C. The mathematical modeling of a novel anchor based on vortex induced vibration. In Proceedings of the OCEANS 2016-Shangha, Shanghai, China, 10-13 April 2016; pp. 1-5. [CrossRef]

15. Pan, F.; Xu, Z.; Pan, P.; Jin, L. Piezoelectric energy harvesting from vortex-induced vibration using a modified circular cylinder. In Proceedings of the 2017 20th International Conference on Electrical Machines and Systems (ICEMS), Sydney, NSW, Australia, 11-14 August 2017; pp. 1-5. [CrossRef]

16. Hai, W.; Chunlai, Y.; Biao, J.; Yanchang, Z.; Gang, Y.; Li, W. Research and Design of Broadband Underwater Flow Induced Vibration Energy Harvester Based on Karman Vortex. In Proceedings of the 2018 IEEE 13th Annual International Conference on Nano/Micro Engineered and Molecular Systems (NEMS), Singapore, 22-26 April 2018; pp. 147-150. [CrossRef]

17. Sun, W.; Su, W. Analysis of a Two-Degree-of-Freedom Piezoelectric Energy Harvester from Vortex-Induced Vibrations. In Proceedings of the 2019 IEEE/ ASME International Conference on Advanced Intelligent Mechatronics (AIM), Hong Kong, China, 8-12 July 2019; pp. 1619-1623. [CrossRef]

18. Yang, X.; He, X. A Piezoelectric Wind Energy Harvester with Interaction Between Vortex-Induced Vibration and Galloping. In Proceedings of the 2019 IEEE SENSORS, Montreal, QC, Canada, 27-30 October 2019; pp. 1-4. [CrossRef]

19. Zhou, G.; Li, Z.; Zhu, Z.; Hao, B.; Tang, C. A New Piezoelectric Bimorph Energy Harvester Based on the Vortex-Induced-Vibration Applied in Rotational Machinery. IEEE/ASME Trans. Mechatronics 2019, 24, 700-709. [CrossRef]

20. Hou, C.; Shan, X.; Zhang, L.; Song, R.; Yang, Z. Design and Modeling of a Magnetic-Coupling Monostable Piezoelectric Energy Harvester Under Vortex-Induced Vibration. IEEE Access 2020, 8, 108913-108927. [CrossRef] 
21. Kundu, A.; Ghosal, D. Influence of Flow Pulsation and Bluffbody on Flow-Induced Elastic Plate Deformation. In Proceedings of the 2020 International Conference on Renewable Energy Integration into Smart Grids: A Multidisciplinary Approach to Technology Modelling and Simulation (ICREISG), Bhubaneswar, India, 14-15 February 2020; pp. 1-4. [CrossRef]

22. ANSYS Inc. ANSYS Fluent User's Guide 17.0. Available online: https:/ / ansyshelp.ansys.com (accessed on 19 December 2017).

23. Catalano, P.; Wang, M.; Iaccarino, G.; Moin, P. Numerical simulation of the flow around a circular cylinder at high Reynolds numbers. Int. J. Heat Fluid Flow 2003, 24, 463-469. [CrossRef]

24. Zdravkovich, M.M. Flow Around Circular Cylinders, Volume 1: Fundamentals; Oxford University Press: New York, NY, USA, 1997; ISBN 978-0-19-856396-9.

25. Warschauer, K.A.; Leene, J.A. Experiments on mean and fluctuating pressures of circular cylinders at cross flow at very high Reynolds numbers. In Proceedings of the International Conference on Wind Effects on Buildings and Structures, Tokyo, Japan, 15-20 August 1971; pp. 305-315.

26. Zdravkovich, M.M. Conceptual overview of laminar and turbulent flows past smooth and rough circular cylinders. J. Wind Eng. Ind. Aerodyn. 1990, 33, 53-62. [CrossRef]

27. Grove, A.S.; Shair, F.H.; Petersen, E.E. An experimental investigation of the steady separated flow past a circular cylinder. J. Fluid Mech. 1964, 19, 60-80. [CrossRef] 